\title{
Is the anti-centromere antibody a marker for a distinct subset of polyautoimmunity in Sjögren's syndrome?
}

\author{
Yun Jong Lee
}

Division of Rheumatology, Department of Internal Medicine, Seoul National University Bundang Hospital, Seoul National University College of Medicine, Seongnam, Korea
Received: October 8, 2021 Accepted: October 18, 2021

\section{Correspondence to \\ Yun Jong Lee, M.D.}

Division of Rheumatology, Department of Internal Medicine, Seoul National University Bundang Hospital, 82 Gumi-ro 173beon-gil, Bundang-gu, Seongnam 13620, Korea Tel: +82-31-787-7049 Fax: +82-31-787-4050 E-mail:yn35@snu.ac.kr https://orcid.org/0000-00017615-8611

\section{See Article on Page 1492-1503}

Sjögren's syndrome (SS) is a systemic autoimmune disease characterized by sicca symptoms (a major clinical feature) and B cell dysregulation and hyperactivity (major pathogenic mechanisms); among autoimmune diseases, it has the highest risk of non-Hodgkin's lymphoma (NHL). Unlike systemic lupus erythematosus (SLE) and rheumatoid arthritis (RA), the clinical course of SS is not characterized by intermittent relapses; instead, it has a stable or very slowly progressive course. Therefore, the diagnosis of SS is often delayed for several years and many individuals remain undiagnosed [1].

Although subjective sicca is the symptom most likely to lead to a diagnosis of SS, SS can have a wide range of extra-glandular manifestations [2]. Moreover, SS patients frequently have polyautoimmunity ( $4 \%$ to $30 \%$ ) overlapping with RA, SLE, systemic sclerosis $(\mathrm{SSc})$, or primary biliary cholangitis (PBC) [3]. The term primary SS is typically applied to cases with SS alone, while secondary SS has been used to describe cases in which another well-defined rheumatic disease is also present. However, the concept of secondary SS may decrease awareness of the presence of SS, thus leading to its underestimation, and can result in an erroneous diagnosis of SLE or RA $[1,4]$. The nomenclature of primary and secondary SS is controversial and does not have significant clinical implications for SS management; therefore, the US Sjögren's Foundation advises changing the terminology [4]. Consequently, the diagnosis of SS in routine practice is challenging in some cases.

Although it is still not known whether autoantibodies have a direct pathogenetic role or merely constitute an epiphenomenon, SS patients exhibit a broad range of organ-specific and -nonspecific autoantibodies secondary to polyclonal $\mathrm{B}$ cell activation. Organ-nonspecific anti-nuclear antibodies (ANAs), rheumatoid factor, anti-Ro/SSA, and anti-La/SSB were long considered diagnostic markers for SS. However, the presence of anti-Ro/SSA or focal lymphocytic sialadenitis with a focus score $\geq 1$ is mandatory for diagnosing SS according to recent criteria [2]. Other autoantibodies tend to be associated with a clinical phenotype, including cryoglobulins, anti-cyclic citrullinated peptide antibodies, and anti-centromere antibodies (ACAs).

ACAs are a type of ANA; they are autoantibodies against centromere-asso- 
ciated proteins (CENPs). ACA positivity is usually determined by a discrete speckled pattern in HEp-2 cells in indirect immunofluorescent (IIF) assays. ACAs react with various components of the centromere-specific nucleosome, the kinetochore. Antigenic proteins include CENP-A, CENP-B, CENP-C, chromobox protein homo$\log 5$ (CBX5), and MIS12 kinetochore complex component (MIS12C) [5]. Of these, anti-CENP-B is considered the main autoantigen because anti-CENP-B positivity in solid-phase assays is strongly associated with the presence of ACAs on IIF assays. ACA is an autoantibody found in SSc patients, especially in a limited cutaneous subset, and is also detected in the autoimmune diseases SS and PBC. According to several recent papers, the prevalence of ACA in East Asian SS patients ranges from $8.2 \%$ to $25.3 \%$ (Table 1) [5-11]. Since the rate of ACA positivity in SSc was $25.5 \%$ in a large Korean multicenter study [7], a considerable proportion of SS patients have ACAs. A retrospective Japanese study reported that 81 (37.9\%) of 309 ACA-positive patients were ultimately diagnosed with SSc, and 50 (23.4\%) with SS [12]. Furthermore, it was recently reported that ACA-secreting cells accumulate in the salivary glands of SS patients [13]. Therefore, researchers have been examining the clinical characteristics of ACA-positive SS.

ACA-positive SS patients have more severe symptoms and signs of exocrine dysfunction, and higher prevalences of Raynaud's phenomenon and sclerodactyly [13]. Autoimmune responses with autoantibodies occur in genetically susceptible individuals, and the associated diseases evolve progressively in a multi-hit fashion. Raynaud's phenomenon and ACA can predict the subsequent development of SSc. Valentini et al. [14] reported that SSc developed in 52.3\% of subjects with serological
(SSc-specific autoantibodies) and capillaroscopic markers, and in $66.6 \%$ of those with serological markers, when individuals with Raynaud's phenomenon were followed for a median 3 years. Several studies have also revealed that ACA-positive SS patients can progress to overt SSc [15]. Therefore, ACA is a risk factor for the subsequent occurrence of overt SSc in SS patients. However, the progression rate ranged widely, from o\% to $40 \%$ [6], likely due to differences among studies in cohorts, sample sizes, and definitions of SS and SSc. Because many patients with ACA-positive SS do not progress to SSc, ACA appears to be a serological marker of a distinct intermediate condition between SS and SSc.

In this issue of the Korean Journal of Internal Medicine, Park et al. [16] report on the clinical features of Korean SS patients with ACA. In the Korean Initiative of primary Sjögren's Syndrome (KISS), ACA was positive in 16.7\% (53/318) of patients when assessed using a commercial enzyme-linked immunosorbent assay. This cross-sectional study is the largest to investigate the impact of ACA positivity on the manifestations of Korean SS patients. At the time of study enrollment, the ACA-positive group was older; it also had higher rates of Raynaud's phenomenon (49.1\% vs. $15.2 \%$ ) and hepatic involvement (11.3\% vs. $0.8 \%$ ), and lower rates of articular involvement (32.1\% vs. $47.7 \%)$, hypergammaglobulinemia (29.4\% vs. $50.4 \%)$, and leukopenia (17.3\% vs. $33.5 \%)$. However, ACA-positive and negative SS groups had comparable glandular dysfunction, disease activity, damage scores, and quality of life indices. Concerning symptoms associated with ACA, the results concur with those of previous studies, including another Korean study [6].

However, some points need to be addressed in Park et al. [16]. First, $32(61.5 \%)$ of their 52 ACA-positive SS

Table 1. Prevalence of anti-centromere antibody in East Asian patients with SS and SSc in the recent literature

\begin{tabular}{lcccc}
\hline Study & Country & Publication year & SSc & SS \\
\hline Lee et al. [6] & Korea & 2015 & & $13 \cdot 4 \%(\mathrm{n}=82)$ \\
Moon et al. [7] & Korea & 2018 & $25.5 \%(\mathrm{n}=751)^{\mathrm{a}}$ & \\
Tsukamoto et al. [8] & Japan & 2018 & $8.2 \%(\mathrm{n}=585)$ \\
Takeshita et al. [5] & Japan & 2020 & $34 \%(\mathrm{n}=35)$ & $13 \%(\mathrm{n}=112)$ \\
Lin et al. [9] & China & 2021 & $13.5 \%(\mathrm{n}=333)$ \\
Lee et al. [10] & Korea & 2021 & $48.4 \%(\mathrm{n}=62)$ & $13.6 \%(\mathrm{n}=59)$ \\
Nakamura et al. [11] & Japan & 2021 & & $25.3 \%(\mathrm{n}=87)$ \\
\hline
\end{tabular}

SS, Sjögren's syndrome; SSc, systemic sclerosis.

aThe number of study subjects. 
patients were positive for both ACA and anti-SSA/Ro. Unfortunately, the authors did not analyze the clinical features after further stratification based on the ACA and anti-Ro/SSA status. The double anti-Ro/SSA- and ACA-positive subgroup may dilute or alter some characteristics of the ACA-positive SS patients. Suzuki et al. [15] reported that anti-Ro/SSA and ACA double-positive SS is also different from anti-Ro/SSA and ACA single-positive SS. Second, Park et al. [16] did not specify the details of the immobilized antigen in the ELISA assay that they used. Several studies of epitope specificity have demonstrated that anti-CBX 5 and anti-CENP-C are more common in SS than SSc patients [5]. However, a recent study of autoantigen profiling of ACA showed similar reactivities against centromere antigens in SS and SSc, unlike previous reports, and ACA-positive SS, SSc, and PBC patients had common clinical characteristics [13]. A further study should investigate if Korean SS patients with ACA have distinct epitopes from SSc and PBC patients with ACA. Third, Park et al. [16] did not examine the longterm outcome of the ACA-positive SS group; however, because their study was based on the KISS prospective cohort, it will be possible to determine how many subjects progress to SSc or develop NHL during follow-up.

In conclusion, this large, well-organized study has important implications for clinicians dealing with ACA-positive results. ACA-positive Korean patients should not automatically be interpreted considered a limited subtype of SSc, because ACA may also be detected in a subset of SS or PBC that shares some clinical features of SSc. In addition, a minor salivary gland biopsy is strongly recommended to facilitate the diagnosis of SS in ACA-positive and anti-Ro/SSA-negative cases suggestive of SS.

\section{Conflict of interest}

No potential conflict of interest relevant to this article was reported.

\section{REFERENCES}

1. Rasmussen A, Radfar L, Lewis D, et al. Previous diagnosis of Sjögren's syndrome as rheumatoid arthritis or systemic lupus erythematosus. Rheumatology (Oxford) 2016;55:1195-1201.
2. Shiboski CH, Shiboski SC, Seror R, et al. 2016 American College of Rheumatology/European League Against Rheumatism classification criteria for primary Sjogren's syndrome: a consensus and data-driven methodology involving three international patient cohorts. Arthritis Rheumatol 2017;69:35-45.

3. Anaya JM, Rojas-Villarraga A, Mantilla RD, Arcos-Burgos M, Sarmiento-Monroy JC. Polyautoimmunity in Sjogren syndrome. Rheum Dis Clin North Am 2016;42:457-472.

4. Kollert F, Fisher BA. Equal rights in autoimmunity: is Sjogren's syndrome ever 'secondary'? Rheumatology (Oxford) 2020;59:1218-1225.

5. Takeshita M, Suzuki K, Kaneda Y, et al. Antigen-driven selection of antibodies against SSA, SSB and the centromere 'complex', including a novel antigen, MIS12 complex, in human salivary glands. Ann Rheum Dis 2020;79:150-158.

6. Lee KE, Kang JH, Lee JW, et al. Anti-centromere antibody-positive Sjogren's syndrome: a distinct clinical subgroup? Int J Rheum Dis 2015;18:776-782.

7. Moon KW, Lee SS, Lee YJ, et al. Clinical and laboratory characteristics and mortality in Korean patients with systemic sclerosis: a nationwide multicenter retrospective cohort study. J Rheumatol 2018;45:1281-1288.

8. Tsukamoto M, Suzuki K, Takeuchi T. Clinical and immunological features of anti-centromere antibody-positive primary Sjogren's syndrome. Rheumatol Ther 2018;5:499505 .

9. Lin W, Xin Z, Ning X, et al. Clinical features and risk factors of Raynaud's phenomenon in primary Sjogren's syndrome. Clin Rheumatol 2021;40:4081-4087.

10. Lee KA, Choi W, Kim J, Kim HS. High prevalence of salivary gland ultrasound abnormalities in systemic sclerosis. Joint Bone Spine 2021;88:105113.

11. Nakamura H, Morimoto S, Shimizu T, Takatani A, Nishihata SY, Kawakami A. Clinical manifestations in anti-Ro52/SS-A antibody-seropositive patients with Sjogren's syndrome. Immunol Med 2021 May 14 [Epub]. https://doi. org/10.1080/25785826.2021.1919342.

12. Tsukamoto M, Suzuki K, Takeuchi T. Initial presentation determines clinical entity in patients with anti-centromere antibody positivity. Int J Rheum Dis 2019;22:103107.

13. Kajio N, Takeshita M, Suzuki K, et al. Anti-centromere antibodies target centromere-kinetochore macrocomplex: a comprehensive autoantigen profiling. Ann Rheum Dis 
2021;80:651-659.

14. Valentini G, Marcoccia A, Cuomo G, et al. Early systemic sclerosis: analysis of the disease course in patients with marker autoantibody and/or capillaroscopic positivity. Arthritis Care Res (Hoboken) 2014;66:1520-1527.

15. Suzuki Y, Fujii H, Nomura H, et al. Impact of double positive for anti-centromere and anti-SS-a/Ro antibodies on clinicopathological characteristics of primary Sjogren's syndrome: a retrospective cohort study. Mod Rheumatol 2018;28:872-878.

16. Park Y, Lee J, Koh JH, et al. Clinical influences of anticentromere antibody on primary Sjogren's syndrome in a prospective Korean cohort. Korean J Intern Med 2021;36:1492-1503. 